\title{
Labyrinthe
}

$32 \mid 2009$ (1)

Le petit théâtre intellectuel

\section{Généalogie de la docilité dans l'Antiquité et le haut Moyen Âge, Gaëlle JEANMART}

\section{Sévérin Yapo}

\section{(2) OpenEdition}

12 Journals

Édition électronique

URL : http://journals.openedition.org/labyrinthe/4014

DOI : $10.4000 /$ labyrinthe.4014

ISSN : 1950-6031

Éditeur

Hermann

\section{Édition imprimée}

Date de publication : 19 juin 2009

Pagination : 177-185

ISBN : 978-2-7056-6885-3

\section{Référence électronique}

Sévérin Yapo, "Généalogie de la docilité dans l'Antiquité et le haut Moyen Âqe, Gaëlle JEANMART »,

Labyrinthe [En ligne], 32 | 2009 (1), mis en ligne le 01 février 2011, consulté le 20 avril 2019. URL:

http://journals.openedition.org/labyrinthe/4014; DOI : 10.4000/labyrinthe.4014

Propriété intellectuelle 


\section{GÉNÉALOGIE DE LA DOCILITÉ \\ DANS L'ANTIQUITÉ \\ ET LE HAUT MOYEN AGE \\ de Gaëlle JEANMART ${ }^{1}$}

Sévérin YAPO

yapson7@yahoo.fr

Les « systèmes pédagogiques chrétiens » (p. 93) débutent en 376. Ils parviennent à accompagner le pouvoir séculier dans son souci de prémunir la société occidentale de la culture païenne, distillée par les invasions ayant déferlé sur l'Occident et entraîné, en 476, la chute de l'Empire romain, parce qu'au plus fort des invasions, les monastères sont restés les seuls foyers d'études. Par la suite, lorsque clercs et moines se donnent pour charge d'éduquer une Europe constellée de mondes païens, c'est dans un dessein bien précis, celui de l'Eglise en lutte contre la « brillante civilisation romaine », de s'allier ces « peuplades barbares » (p. 94). Le projet chrétien s'accompagne ainsi de la culture d'un véritable « art de la servitude » (p. 9). Plusieurs siècles après, les choses ne semblent pas avoir beaucoup évolué, au niveau pédagogique à tout le moins. C'est de l'hégémonie, toujours actuelle, de l'idéologie des pratiques pédagogiques du Moyen Âge, que Gaëlle Jeanmart se désole.

À ses yeux, l'idéologie monastique a perverti la nature humaine. Jeanmart pose donc la question suivante: saurait-on encore accompagner vers la liberté des êtres dont la nature est la raison, si tant est que tout acte d'éducation semble soumis aux liens de l'obéissance - la docilité - et de l'autorité - signe de pouvoir - (p. 7) ? Une alternative est-elle possible? Jeanmart se propose de répondre par l'affirmative à une telle question, en faisant référence au paradigme grec de la pédagogie, illustré par l'éthique pédagogique privée de Socrate, et à sa politisation par Aristote, grâce au sens commun. C'est même ce retour à l'origine de nos pratiques éducatives actuelles, où elle rencontre l'expérience grecque antique de

1. Paris, Vrin, 2007, 272 p., $30 €$. 
la docilité, qui rend Jeanmart attentive à l'inflexion décisive subie par cette notion chez les moines chrétiens, dès le haut Moyen Âge.

Son ouvrage, traversé par la quête du « courage de la vérité ${ }^{1}$, ce qui témoigne de l'inscription de son auteur dans la lignée des cours de Foucault sur le gouvernement de soi et des autres, comprend deux parties. La première présente " la notion de docilité dans le système éducatif grec " (pp. 18-92), et analyse d'abord " la parrhèsia socratique » (pp. 18-57) puis «Aristote ou la liberté de définir et de réaliser sa nature propre » (pp. 57-92). La deuxième partie est consacrée à « la notion de docilité dans les monastères du haut Moyen Âge » (pp. 93-247). L'attention de l'auteur se focalise sur « l'esprit de la formation monastique » (pp. 93-154), sur la matérialisation de cet esprit à travers « les Institutions cénobitiques de Cassien » (pp. 154-208) et « la règle de Saint Benoît et celle du Maître » (pp. 208-247).

Dans la première partie du livre, l'auteur étudie la vision socraticoaristotélicienne de l'éducation, autrement dit « l'art de conduire à la liberté des êtres assujettis à leurs passions » (p. 9). Elle examine comment cet art privé chez Socrate est érigé en science par Aristote, grâce au sens commun, à la délibération et à la prudence. S'intéressant d'abord à « la parrhèsia socratique », elle oppose la parrhèsia (le dire vrai) à un autre concept: l'elenchos (la réfutation), ce qui lui permet de rediscuter les liens de Platon et d'Aristote à Socrate. À la racine, la parrhèsia - de pan (tout) et rhema (ce qui est dit)-, renvoie au fait de tout dire. Caractérisant la philosophie pratique dans son rapport à la manière de vivre et à la vérité ${ }^{2}$ la parrhèsia induit la possibilité de modifier l'être et l'éthos de l'auditeur par la radicalité du dire vrai qu'elle recèle, sans égard pour quelque intérêt sophistique qui pourrait incliner à user de rhétorique. Or, l'elenchos, défini par Platon comme réfutation ${ }^{3}$, s'avèrera incapable de répondre au dessein éthico-pratique du dire vrai. En s'appuyant sur la position de Brian Vickers et John Beversluis, qui jugent que le « Socrate platonicien ne vise jamais tant le progrès moral et intellectuel

1. Michel Foucault, Le gouvernement de soi et des autres, Paris, Seuil, Gallimard, 2008.

2. Julius Domanski, La philosophie, théorie ou manière de vivre?, Paris, Fribourg, Le Cerf, Editions Universitaires de Fribourg, n 18, 1998, pp. 20-29.

3. Platon, Sophiste, 230 b, traduction française de Nestor Cordero, Paris, Garnier-Flammarion, 1993, p. 108. 
de ses interlocuteurs [...]» (p. 20), Jeanmart en vient ainsi à rapprocher davantage Aristote de Socrate, en prenant le parti de Xénophon pour qui « l'elenchos ne saurait être un moyen d'éducation morale, contrairement à ce qu'enseigne la doctrine du Sophiste » (p. 20) de Platon. En arguant finalement, avec Louis-André Dorion, que « Platon lui-même a finalement délaissé l'elenchos et qu'Aristote s'est empressé d'amputer la réfutation de toute visée éthique ${ }^{1} »$, Jeanmart soutient que la parrhèsia est plus typique du philosopher socratique que l'elenchos, auquel on a voulu le limiter. Pour elle, l'avantage de la parrhèsia sur l'elenchos ne tient pas à l'extériorité possible d'un propos, dont l'efficace est édulcorée par la volonté de piéger, mais réside dans la positivité d'une praxis, exposant son acteur au sérieux de l'espace relationnel et communautaire, avec le risque de mettre en péril sa vie en déplaisant. Ce courage inhérent à la parrhèsia socratique constitue le premier lieu de réconciliation possible entre Platon et Aristote. Jeanmart observe en effet que tant les Mémorables de Xénophon que le Lachès de Platon définissent le courage comme une science objective qui discrimine les possibles situationnels (p. 27), mais également comme une science fondamentale des valeurs et principes imposés par le sujet à soi-même comme signature de son entière liberté.

Elle fait ainsi culminer l'éminence de la dimension éthique du geste parrhèsiaste dans le procès de Socrate, dont la vie a consisté en la quête permanente de la conformation de l'agir au dire, un dire identique au penser du sujet. Socrate ne cherche pas à plaire. C'est ce dire risqué de la vérité qui le rend plus apte à réussir la mission délicate de l'éducation citoyenne: rendre libre. Mais Socrate ne se réfère qu'à son daimon intérieur, par où il se ferme à sa communauté. Ce savoir éthique que Socrate communique en privé, sans pour autant faire profession de pédagogue, Aristote entreprend d'en faire une science nomothétique, partageable dans la communauté des hommes; science que Jeanmart, en l'absence de traces textuelles suffisantes, explicite ensuite, en s'appuyant sur la pensée politique d'Hannah Arendt.

L'auteur base ainsi le décryptage du système pédagogique aristotélicien sur la théorie de l'esclavage et de la liberté que recèle la pensée

1. Xénophon, Mémorables, tome I, texte établi par Michèle Bandini et traduit par Louis-André Dorion, Paris, Les Belles Lettres, Collection Budé, 2000, Introduction générale, Livre I, p. CXLII. 
aristotélicienne. Pour Aristote, l'esclavage est l'état premier de tous. Comme la psychologie platonicienne, la psychologie aristotélicienne reste sensible à la nécessité d'une soumission de la partie appétitive de l'âme à sa partie rationnelle. Le sujet est entraîné à orienter ses plaisirs, d'abord esclaves, vers les œuvres qui élèvent et font la joie de l'esprit d'un être dont la nature advenue est liberté, une liberté appelée à délibérer dans l'assemblée des hommes. L'obéissance, apprise par accoutumance, à soumettre sa chair à la force de la volonté, devient donc peu à peu rationnelle, intériorisée qu'elle est via l'assujettissement passager au maître. Celui-ci n'a autorité que pour autant qu'il est porteur d'un savoir pratique éprouvé par le temps; il est surtout un moyen, le serviteur d'une raison en phase d'autonomisation chez le disciple mais, par essence, déjà partagée avec ce dernier, dont la subordination se muera en s'achevant comme maîtrise de soi. En commandant à l'élève, l'éducateur reconduit sociétalement ici ce que le parrhèsiaste socratique réalisait en son être personnel: à savoir le principe selon lequel seule la raison oblige.

Parce que, pour Aristote, vouloir c'est pouvoir, point n'est besoin d'espérer de fins qui échapperaient à la maîtrise de l'homme. D'où la part belle faite à la délibération. Celle-ci portera surtout sur les moyens de vivre bien et non point de vivre éternellement, par exemple, vu qu'espérer vivre éternellement ne contient point le pouvoir d'une telle éternisation du vivre. Par là, Jeanmart fait de l'entreprise d'Aristote l'accomplissement politique de l'éthique de Socrate, Aristote s'étant astreint à l'exigence d'une méthode d'éducation qui engage toute la communauté des hommes. Cette communauté ne réunit plus des besoins économiques - déjà résolus dans la stricte cellule familiale, où l'autorité monarchique du père s'est justifiée dans sa fonction de pourvoyeur - mais plutôt le partage d'un sentir en commun. Elle est le lieu d'amitié entre pairs. Pour ces derniers, le faire prime sur l'apprendre, en tant que l'épreuve de soi au feu de l'expérience toujours particulière et, qui plus est, ouverte à la délibération, a préséance, pour ce qui est des affaires des hommes, sur le savoir théorique par trop généralisant des philosophes spéculatifs. La finalité de l'obéissance est d'être à même de se déterminer soi-même en regard des autres, avec qui l'on vit. Ce vivre ensemble dit la construction concertée de la cité par des êtres qui réalisent continûment le partage de leur commune nature: une liberté vécue. 
Cet exposé jeanmartien du système grec a pour effet de faire la lumière sur le désordre rationnel de la pédagogie monastique du Moyen Âge, qui sous-tend une communauté religieuse, et dont le dispositif est focalisé sur le seul combat contre la chair, auquel cette pédagogie finit par asservir insidieusement le moine. Là où les moines enseignent « un contenu de doctrine » (p. 9), les Grecs proposent « une attitude de vie », le « choix éthique d'un système de valeur» (p. 9). Il s'agit pour ceux-ci de « transmettre à un individu la vertu par laquelle il réalisera le plus son humanité » (p. 9). Ce point est d'importance. Il permet à l'auteur de montrer que l'éducation monastique est un travestissement de l'idéal grec d'accomplissement du sujet, une vaste entreprise d'assujettissement d'une humanité désubstantialisée et dont la modernité occidentale est l'inconsciente héritière. Par où apparaît l'accent nietzschéen du texte, qui est sous-jacent dans cette critique de la subordination insoupçonnée de l'éducation à des manœuvres politiciennes, illustrée par les us propres aux institutions monastiques. Qu'en est-il précisément?

L'auteur, dans la seconde partie de son ouvrage, intitulée « la notion de docilité dans les monastères du haut Moyen Âge », critique la pédagogie chrétienne. De celle-ci, elle commence par expliciter les règles. Restituant « l'esprit de la formation monastique », Jeanmart rend compte des fondements à partir desquels les chrétiens balaieront progressivement toutes les cultures païennes antérieures, en installant le monachisme de leur modèle éducatif propre. Il s'agit en particulier de l'ascèse (érémitisme) désertique (anachorétisme), qu'ils rendront communautaire (cénobitisme) sous l'idée de l'Institution comme corps aux pratiques minutieusement réglementées. Dans ce cadre, les moindres instants des journées du moine sont ainsi occupés. La Bible est exaltée comme unique source de culture. Sa lecture psalmodiée et ruminée hante l'âme du moine si le travail manuel ne l'a pas encore abruti. L'exaltation de la docilité vise à pousser l'apprenant à renoncer à son œuvre propre au profit de l'œuvre de Dieu. L'exemple d'obéissance du Christ est alors brandi, cependant qu'ici il ne faut obéir qu'à un homme: le curé, en l'occurrence.

Qu'implique pour le citoyen le fait que les pratiques éducatives occidentales aient une origine monacale? En étudiant cette question, Jeanmart tente de rejoindre les replis de la personnalité de chaque moi moderne dont elle veut interroger la posture face aux modes actuels 
d'enseignement. Elle décrit les affres voilées d'une vision mythologique, là où l'homme est conçu comme déchu du fait du péché d'Adam, prétendument responsable de l'irréversible décadence de la volonté d'un certain sujet humain. Consciente de cette réduction de l'ego du sujet à une volonté, condamnée à la corruption, Jeanmart décèle dans l'argument de la reconquête d'une consistance ontologique de l'homme, l'incohérence de la finalité téléologique de l'éducation chez les chrétiens. Le chemin de retour entrevu par ces derniers est l'obéissance dite réparatrice d'un homo interior (p. 123), malade, et qu'il faudrait désormais tenir hors de tout monde - y compris le sien propre, bizarrement !

Jeanmart dérive du grec monachos - uni -, la tradition chrétienne du monachus - « qui vit seul (ermite ou anachorète) -, celui qui a renoncé à sa famille, au mariage, à ses biens; celui qui vit dans l'unité avec d'autres (cénobite) [...] et enfin celui qui est unifié, le simple» (p. 121). Cet isolement factuel est le produit de l'éducation moyen âgeuse. Dans la « cité » des moines, étrangement policée, et qui fonctionne comme une famille, seul le curé a le pouvoir. Décidant pour tous, il revendique la responsabilité du devenir éternel de chacun des membres de la « cité ». Le sort collectif, maintenu par la peur, est perçu comme pouvant déchoir par la faute d'un seul. Le curé impose ainsi l'absolu d'un impératif de transparence qui coupe, pour l'individu, jusqu'à la possibilité de détenir quelque bien matériel ou intellectuel. Prétextant l'omniprésence de la tentation, on amène le disciple à se river à sa seule chair. Si par mégarde, ou fatigue, il flanche, une punition exemplaire lui est administrée. Châtiant l'esprit sous prétexte de discipliner la chair, on abrutit le sujet. Par ailleurs, exaltant un Christ humilié, on tue l'amour d'un Dieu vivant. Celui-ci fait l'objet d'un deuil antigonien: sans sépulture ni consolateurs, ni même espace pour l'exprimer. En guise de psychanalyse impossible à accomplir, Jeanmart révèle chez le moine le scellement des forces de vie. Le Dieu aimé ne pouvant être enterré, le deuil est deuil de la foi en cet aimé. La foi véritable apparaît ainsi comme l'ennemi d'une entreprise de négation de l'homme.

Quels liens y a-t-il entre le tableau ci-dessus et notre état social actuel? Les deuxième et troisième chapitres de cette deuxième partie, qui exposent une espèce de descente dans les deux laboratoires d'expérimentation de la docilité collective dont naîtra la moderne organisation pédagogique, invitent à soulever une telle question. D'abord, dans son 
chapitre sur « les Institutions cénobitiques de Cassien », Jeanmart montre comment Cassien, par l'imposition de ses règles en Occident, a fermé toutes possibilités d'accès à l'idiorythmie, caractéristique du monachisme oriental, d'êtres disposant à leur guise de leur temps, de leur vie donc. Ensuite, dans « la règle de Saint Benoît et celle du Maître », convenant, avec Foucault, que « la qualification individuelle, intellectuelle ou morale ${ }^{1} »$, est le seul critère valide pour une prétention à des fonctions de responsabilités sociales, Jeanmart met le doigt sur le pouvoir social du «pastorat» des bergers modernes, dont elle s'étonne de l'infondé éthique. Là où les Grecs valorisaient le courage, les chrétiens se drappent des oripeaux de la docilité, par peur de s'autonomiser en s'engageant d'eux-mêmes pour leur bien. À la pédagogie comme science du public, le chrétien substitue une connaissance de l'individu dans le détail de sa personnalité afin de mieux le manipuler. Le dernier lieu de retrait de toute portée politique à l'œuvre monastique apparaît lorsque Jeanmart réfléchit sur le souci de soi. Selon elle, quand les revendications sont strictement individuelles, l'individualisme croît et s'achève dans la réclusion qu'aura précédé la fermeture de l'univers monacal à tout souci d'évangélisation du voisinage, au nom d'une identité collective de façade à préserver. La stérilité du projet éducatif monastique atteint donc son faîte dans l'absence d'objectif commun de construction d'un monde, visée qui aurait pu fédérer les compétences particulières d'êtres unis et égaux.

Ce livre a le mérite d'orienter le débat contemporain sur l'éducation et d'inviter à faire émerger un système plus libéré de l'héritage du Moyen Âge, lequel a dépolitisé l'idéal éthique grec. Chaque fois qu'elle présente une pratique chrétienne, Jeanmart prend le soin de l'éclairer de son contraire antique. En plus de traduire des sources grecques, allemandes ou latines lorsqu'il le faut, elle sait dépasser l'ambition d'une anthropologie atemporelle à la Durkheim, d'une approche de «l'homme dans sa totalité ${ }^{2} »$ (p. 14). Elle interroge « la contingence à l'œuvre» dans les " pratiques et les discours » expressifs des « relations de maîtrise » inhérentes aux pratiques éducatives spécifiques à l'" Antiquité et au Moyen Âge » (p. 14).

1. Michel Foucault, «Sexualité et pouvoir », in Dits et Écrits, Paris, Gallimard, 1994, p. 560.

2. Emile Durkheim, L'évolution pédagogique en France, Paris, PUF, 1969, p. 7. 
Radicalisant l'étonnement, dans une perspective nietzschéenne, Jeanmart est passée du « d'où venons-nous? » au « qu'est-ce que notre héritage a fait de nous? ». Elle dépeint ainsi l'issue de l'esprit de bien des pratiques modernes courantes, telle l'errance loin de soi, qui préside à l'hypocrisie d'une codification outrée de la politesse. En notre actualité de déperdition des valeurs, la morale est « identifiée [...] à la politesse » (p. 208). « Humains trop humains », pour reprendre le titre d'un ouvrage de Nietzsche, sans intériorité ni projet réfléchi et partagé de construction d'une humanité ensemble, la majorité d'entre nous a été encouragée, au nom d'un pseudo « oubli de soi» (p. 206), typique des Institutions cénobitiques de Cassien, à remettre le « souci de soi » socratique entre les mains de bergers anonymes, gouvernants ou pasteurs, par suite de la désertion des nomothètes aristotéliciens. Et comme l'homme ne peut vivre sans mythe, on a fini par persuader cette masse rivée à la plate superficie des choses, qu'être moral, c'est faire un bon étalage de la mimique des « usages particuliers définis par l'institution comme adéquats » (p. 208), usages à travers lesquels on poursuit un spectre d'esprit ${ }^{1}$. Très critique à ce sujet, Jeanmart s'inscrit, pour sa part, dans une démarche d'appropriation et de progrès. Respectueux de la relation au cénobitisme oriental et incluant la question du pouvoir, son livre est peut-être un pas décisif vers l'aurore de systèmes ou de pratiques éducatifs typiquement contemporains. Car la question de " la communauté », qui « nous hante, nous déserte ou nous embarrasse ${ }^{2} »$, face aux risques d'un nivellement déshumanisant à l'ère de la mondialisation, exige, de la part des spécialistes de la formation et des gouvernants, l'intelligence d'un " accompagnement ${ }^{3}$ » efficient vers la construction d'un Soi, tant personnel - exercé au nom

1. Pour un philosophe comme Heidegger, en pareille situation, l'unique but de la pensée sera de « maîtriser le danger d'obscurcissement du monde » et de parvenir à « un réveil de l'esprit » (Martin Heidegger, Introduction à la métaphysique, traduction française de Gilbert Kahn, Paris, Gallimard, 1967, p. 61).

2. Jean-Luc Nancy, La communauté affrontée, Paris, Galilée, 2001, p. 26.

3. Le concept d'accompagnement renvoie, pour $\mathrm{M}$. Beauvais, à une « démarche visant à aider une personne à cheminer, à se construire, à atteindre ses buts » (Martine Beauvais, « Des principes éthiques pour une philosophie de l'accompagnement », dans De l'éducation permanente à la formation tout au long de la vie, Savoirs, ${ }^{\circ}$ 6, Paris, L’Harmattan, 2001, p. 101). 


\section{du « courage de la vérité ${ }^{1} »-$, que communautaire, - par un certain stoïco-aristotélicisme ${ }^{2}$.}

1. Tel le «fou » du roi, le soi «parrhèsiaste » est capable, « contre la folie, contre l'aveuglement » possible « du maître », de dire la vérité: il « se dresse, [...] va dire le vrai, et par conséquent limiter par là la folie du maître» (Michel Foucault, op. cit., p. 148). Sa démarche, cependant, peut ne pas engager toute la communauté.

2. L'enjeu n'est-il pas de faire advenir un monde, une communauté de " parrhèsiastes »? Le stoïcisme politique d'accentuation aristotélicienne que recèle entre autres thèmes le texte de Danielle Lories, $L e$ sens commun et le jugement du phronimos, Aristote et les Stoïciens, Louvain-la-Neuve, Peeters, 1998, et auquel fait d'ailleurs allusion Jeanmart (p. 90), gagnerait à être approfondi à cet effet. Il s'agirait de discuter les positions de Foucault, pour qui la philosophie n'a pas vocation à s'occuper de « la justice et de l'injustice dans la cité » (op. cit., p. 295) mais plutôt de « la question du sujet dans la politique » et donc des seules conditions pour que le parrêsiaste, en posture de « souverain », sache éviter d'« être l'agent lui-même de l'injustice» (ibidem., p. 294). 